\title{
Brote epidémico de dengue en la Ciudad de Buenos Aires, 2016: características clínicas y hematológicas de la infección en una población pediátrica
}

\author{
Dengue outbreak in Buenos Aires, Argentina, 2016: Clinical and hematological \\ features in children
}

\author{
Dra. Claudia I. Cazes ${ }^{a}$, Dra. Carolina M. Carballo ${ }^{a}$ Dra. María L. Praino ${ }^{a}$, Dr. Fausto M. Ferolla ${ }^{a}$ \\ Dra. Alicia Mistchenko ${ }^{b}$ Dra. María M. Contrini ${ }^{,}$Dra. Aurelia Fallo y Dr. Eduardo L. López ${ }^{a}$
}

\section{RESUMEN}

El dengue es la arbovirosis humana que más morbimortalidad ocasiona mundialmente. Durante 2016, se registró, en la Ciudad de Buenos Aires, Argentina, la mayor epidemia de esta enfermedad.

Objetivo: describir las características clínicas y hematológicas en una población pediátrica.

Métodos: estudio de corte transversal que incluyó a pacientes atendidos del 18-1-16 al 15-4-16 en el Hospital de Niños "Dr. Ricardo Gutiérrez".

Resultados: se registraron 156 casos, 82 confirmados por virología; $130(83 \%)$, autóctonos. Las manifestaciones clínicas más frecuentes fueron fiebre, cefalea y dolor retroocular. Las alteraciones del laboratorio significativas fueron leucopenia, plaquetopenia y aumento de transaminasas. Se internaron 35 pacientes $(23 \%), 25(16 \%)$ con signos de alarma. No se presentó ningún caso de dengue grave.

Conclusiones: el reconocimiento oportuno de los signos de alarma y el control hematológico resultan fundamentales para detectar a los niños en riesgo y ofrecerles tratamiento de soporte en forma precoz.

Palabras clave: virus del dengue, niño, pediatría.

\section{ABSTRACT}

Dengue is the human arbovirus with the highest morbidity and mortality in the world. The largest outbreak of dengue in Buenos Aires, Argentina, occurred during 2016.

a. Programa de Infectología Pediátrica, Facultad de Medicina, Universidad de Buenos Aires, Hospital de Niños Ricardo Gutiérrez, Ciudad de Buenos Aires, Argentina.

b. Laboratorio de Virología, Hospital de Niños Ricardo Gutiérrez, Ciudad de Buenos Aires, Comisión de Investigaciones Científicas de la Provincia de Buenos Aires (CIC), Argentina.

Correspondencia:

Dra. Claudia Cazes: clauinescazes@gmail.com

Financiamiento: Ninguno.

Conflicto de intereses: Ninguno que declarar.

Recibido: 24-5-2018

Aceptado: 9-8-2018
Objective: To describe clinical and hematological features in children with confirmed dengue infection.

Methods: Cross sectional study that included children attended since January $18^{\text {th }}$ to April $15^{\text {th }} 2016$ at Hospital de Niños "Dr. Ricardo Gutiérrez". Results: among 156 registered cases, 82 confirmed cases by virology test; 130 (83\%) autochthonous cases. The most frequent clinical manifestations were fever, headache and retro-ocular pain.

Laboratory abnormalities were leukopenia, thrombocytopenia and increased liver enzymes. Thirty-five children were hospitalized $(23 \%), 25(16 \%)$ with warning signs. In our study, no cases of severe dengue occurred.

Conclusions: early recognition of warning signs and hematological monitoring is essential in order to detect patients at risk and offer them adequate early treatment.

Key words: dengue virus, child, pediatric.

http:/ / dx.doi.org/10.5546/ aap.2019.e63

Cómo citar: Cazes CI, Carballo CM, Praino ML, Ferolla FM, et al. Brote epidémico de dengue en la Ciudad de Buenos Aires, 2016: características clínicas y hematológicas de la infección en una población pediátrica. Arch Argent Pediatr 2019;117(1):e63-e67.

\section{INTRODUCCIÓN}

Las infecciones por el virus dengue (dengue virus; DENV, por sus siglas en inglés) constituyen la arbovirosis humana que más morbimortalidad ocasiona mundialmente, con un considerable aumento en su incidencia en las últimas décadas. ${ }^{1-3}$ En las áreas no endémicas, los casos podrían subvalorarse, debido a que sus manifestaciones clínicas podrían confundirse con otras enfermedades. ${ }^{4,5}$ En el mundo, se producen, por año, 390 millones de infecciones por DENV, de las cuales alrededor del $25 \%$ se manifiestan clínicamente. ${ }^{6}$ En América, solo Canadá y Uruguay aún no han reportado casos autóctonos. ${ }^{7}$ En 2009, se detectaron los primeros casos de transmisión local en el área metropolitana de Buenos Aires, una zona no endémica, $y$, durante los meses de verano de 2016, se registró el brote más importante hasta la fecha. ${ }^{1,8}$ 


\section{OBJETIVO}

Describir las características clínicoepidemiológicas, de laboratorio y la evolución de los niños con dengue atendidos en un hospital pediátrico de un área urbana no endémica.

\section{MATERIAL Y MÉTODOS}

Estudio de corte transversal que incluyó a todos los pacientes con diagnóstico presuntivo de dengue atendidos en los Consultorios de Infectología y Salas de Internación del Hospital de Niños "Dr. Ricardo Gutiérrez", entre el 18 de enero y el 15 de abril de 2016.

\section{Definiciones}

Caso probable: paciente con antecedente de contacto con un enfermo de dengue o que hizo un viaje a una zona endémica en los 15 días previos a la consulta, acompañado de un cuadro clínico compatible (fiebre de $<7$ días de duración, sin afección de las vías aéreas superiores, con $>2$ de los siguientes síntomas: cefalea y/o dolor retroocular, mialgias, artralgias, exantema, dolor abdominal, náuseas, vómitos, petequias o prueba del lazo positiva y plaquetopenia o leucopenia). ${ }^{2,9}$

Caso confirmado: caso probable con antigenemia (detección del antígeno NS1 mediante ensayo por inmunoabsorción ligado a enzimas [enzyme-linked immunosorbent assay; ELISA, por sus siglas en inglés] o prueba de reacción en cadena de la polimerasa [polymerase chain reaction; $\mathrm{PCR}$, por sus siglas en inglés]) o serología (anti-DENV inmunoglobulina M [IgM] por ELISA) positivas. ${ }^{2,9}$ La serotipificación viral fue realizada mediante PCR. Las técnicas para el diagnóstico virológico utilizadas han sido descritas en estudios previos. ${ }^{1,5}$

Para la clasificación de dengue, dengue con signos de alarma y dengue grave, se utilizaron las definiciones establecidas por la guía del Ministerio de Salud de la Nación Argentina y la Organización Mundial de la Salud. 2,9,10 Se consideró dengue con signos de alarma la presencia de uno o más de los siguientes: dolor abdominal intenso y sostenido; vómitos persistentes; derrame seroso (en el peritoneo, la pleura o el pericardio) detectado por clínica, por laboratorio (hipoalbuminemia) o por imágenes (ecografía de abdomen o radiografía de tórax); sangrado de mucosas; cambio en el estado mental del paciente (somnolencia o irritabilidad); hepatomegalia mayor de $2 \mathrm{~cm}$; incremento brusco del hematocrito concomitante con rápida disminución del recuento de plaquetas. Caso de dengue grave, con uno o más de los siguientes: extravasación grave de plasma, expresada por la presencia de shock hipovolémico y / o por dificultad respiratoria debida al exceso de líquidos acumulados en el pulmón; hemorragias graves; la afectación de órganos: hepatitis grave (transaminasas superiores a 1000 unidades), encefalitis o afectación grave de otros órganos, como la miocarditis.

En cuanto al análisis de laboratorio, se definió leucopenia con un recuento de leucocitos $<4000$ / $\mathrm{mm}^{3}$; neutropenia, recuento de neutrófilos < $1000 / \mathrm{mm}^{3}$; plaquetopenia, recuento de plaquetas $<100000 / \mathrm{mm}^{3}$; aumento de las enzimas hepáticas, transaminasa glutámico oxalacética (glutamic oxaloacetic transaminase; GOT, por sus siglas en inglés) y/o transaminasa glutámico pirúvica (glutamate pyruvate transaminase; GPT, por sus siglas en inglés) $>40 \mathrm{UI} / 1$. Para evaluar el aumento del hematocrito, se analizó el porcentaje de variación durante la evolución de la enfermedad, con respecto al valor basal.

Se utilizó el programa estadístico Stata v13 para el análisis de los datos.

\section{RESULTADOS}

De un total de 1567 consultas ambulatorias, se diagnosticaron $156(9,9 \%)$ casos de dengue, de los cuales $82(52,6 \%)$ fueron confirmados por laboratorio virológico, todos serotipo 1 , y $74(47,4 \%)$ probables, por clínica más nexo epidemiológico.

La mediana de edad fue de 11 años (rango de 4 meses a 21 años). No hubo diferencias en cuanto al género; 78 pacientes (50\%) correspondieron a cada sexo. El 56,4 \%, con residencia en la Ciudad de Buenos Aires, y el 43,6\%, en el Gran Buenos Aires.

De los 156 casos, 130 (83\%) fueron autóctonos, y $26(16 \%)$, importados. De estos, $17(65 \%)$ provenían de Paraguay; 8 (30\%), del Noroeste Argentino, y 1, de Venezuela.

A partir de la semana epidemiológica 6, se evidenciaron los primeros casos autóctonos, que fueron los predominantes a partir de la semana 9 (Figura 1).

Dengue clásico fue la forma de presentación en $130(83,3 \%)$ pacientes; $26(16,7 \%)$ tuvieron dengue con signos de alarma. No se registraron casos de dengue grave. Los signos de alarma consistieron en sangrado de mucosas $(7 / 26$, $26,9 \%)$, dolor abdominal intenso y sostenido $(7 / 26,26,9 \%)$, aumento del hematocrito con disminución del número de plaquetas $(6 / 26$, 
$23 \%)$, vómitos persistentes $(4 / 26,15,3 \%)$, hepatomegalia $(1 / 26,3,8 \%)$ y acumulación clínica de líquidos $(1 / 26,3,8 \%)$.

Se analizaron los casos de dengue confirmado $(\mathrm{n}=82)$ para describir las manifestaciones clínicas: el $100 \%$ de los pacientes presentó fiebre con una mediana de duración de 5 días (rango: 1-9). Se describen las manifestaciones clínicas en la Tabla 1. En el $67 \%$ se presentó una erupción cutánea (Figura 2), solo el 23\% manifestó prurito.

El hallazgo más frecuente de laboratorio fue la leucopenia, que se observó en el $85 \%$ (70/82) de los casos confirmados. El $64 \%$ (53/82) presentó aumento de transaminasas, con una mediana de GOT de 57,5 (rango: 9-619) y de GPT de 35 (rango: 9-338); el $28 \%$ (23/82), plaquetopenia, y el $18 \%$ (15/82), neutropenia.

El 19,5\% (16/82) de los casos confirmados presentó hemoconcentración. En 14 pacientes $(17,1 \%)$, se constató el aumento del hematocrito del 10-19\% con respecto a su valor basal y, en 2 $(2,4 \%)$, se registró un aumento mayor del $20 \%$.

El menor valor de recuento de leucocitos se observó entre el $4 .^{\circ}$ y el $5 .^{\circ}$ día del comienzo de los síntomas, con una mediana de $3100 / \mathrm{mm}^{3}$ (rango: 1300-6200), y la recuperación del valor normal, del $8 .^{\circ}$ al $15 .^{\circ}$ día. Con respecto al recuento de plaquetas, el menor valor se constató entre el $6 .^{\circ}$ y el $7 .^{\circ}$ día de evolución, con una mediana de $150000 / \mathrm{mm}^{3}$ (rango: $21000-300000$ ), y se recuperó el valor normal entre los 8 y los 15 días (Figura 3).

Se internaron 35 pacientes (35/156, $23 \%)$; $25(71,4 \%)$ por presentar dengue con signos de alarma; $8(22,8 \%)$, para el aislamiento entomológico al inicio del brote epidémico, y $2(5,7 \%)$, por presentar comorbilidades ( $1 \mathrm{con}$ virus de la inmunodeficiencia humana -VIH- y 1 con síndrome de hiper-IgE). Ninguno requirió cuidados intensivos. Como complicación, se mencionó un caso de colecistitis alitiásica. La media de duración de internación fue de 2,9 días $( \pm 1,5)$.

\section{DISCUSIÓN}

Según el reporte de la Organización Mundial de la Salud (OMS)/Organización Panamericana de la Salud (OPS), en cuanto al número de casos de dengue y dengue grave en las Américas, en 2016, Argentina informó 76867 casos probables, 41207 casos confirmados por laboratorio de virología y 11 defunciones. ${ }^{8}$

Tabla 1. Manifestaciones clínicas de casos de dengue confirmados $(n=82)$

\begin{tabular}{lc}
\hline Manifestaciones clínicas & N (\%) \\
\hline Cefalea & $69(84)$ \\
Dolor retroocular & $68(82,9)$ \\
Exantema & $55(67)$ \\
Mialgias & $52(63,4)$ \\
Náuseas y vómitos & $42(51,2)$ \\
Dolor abdominal & $42(47,5)$ \\
Prurito & $19(23,1)$ \\
Diarrea & $18(21,9)$ \\
Artralgias & $18(21,9)$ \\
Epistaxis & $15(18,2)$ \\
Petequias & $11(13,4)$ \\
Gingivorragia & $5(6)$ \\
Inyección conjuntival & $3(3,6)$ \\
Poliartritis & $1(1,2)$ \\
Hepatoesplenomegalia & $1(1,2)$ \\
\hline
\end{tabular}

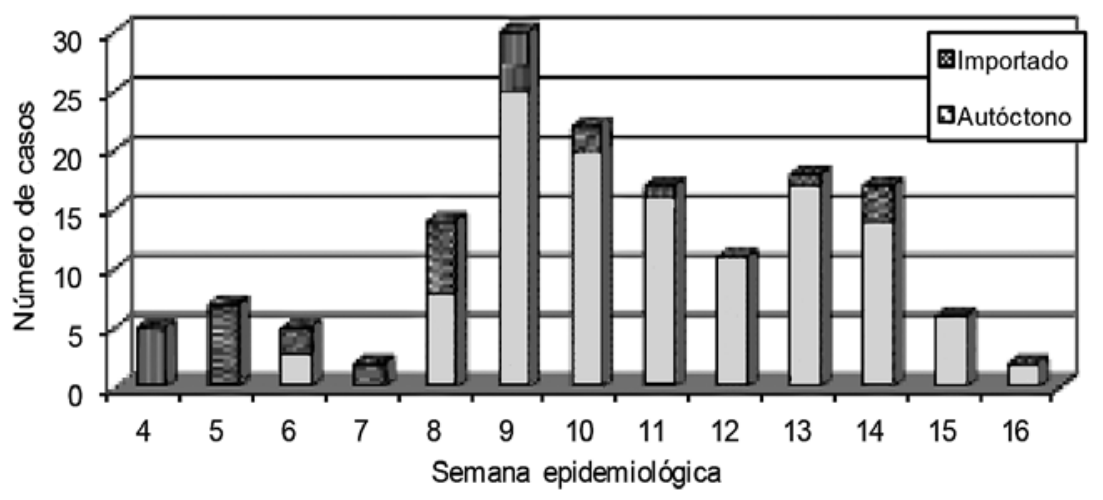


Los primeros casos que se presentaron en nuestro centro eran importados, provenientes de Paraguay en su mayoría (65\%). A partir de la semana epidemiológica 6, se diagnosticaron casos autóctonos, que fueron los que predominaron finalmente y representaron el $83 \%$ del total.

En línea con lo reportado en otros estudios sobre dengue en pediatría, ${ }^{11-14}$ las manifestaciones clínicas más frecuentes en nuestros pacientes fueron fiebre, cefalea, dolor retroocular y mialgias. Aproximadamente, la mitad de los casos presentó síntomas abdominales (náuseas, vómitos, dolor abdominal). Las alteraciones del laboratorio más frecuentes fueron la leucopenia

FIGURA 2. Exantema característico de dengue: signo de "islas blancas en marea roja"
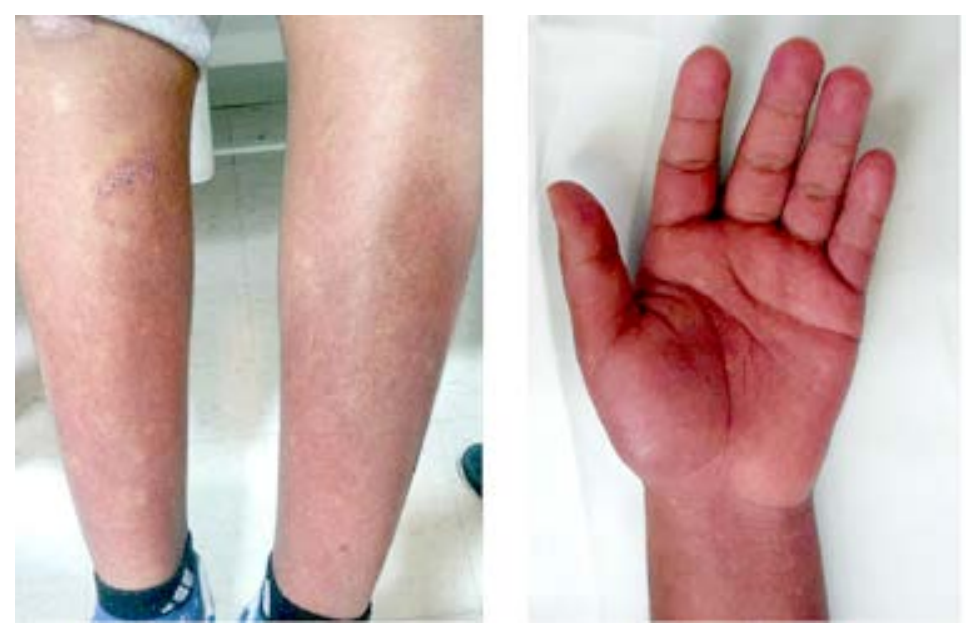

FIGURA 3. Variaciones en el valor de leucocitos y plaquetas según los días de evolución

$\mathbf{a}_{*}$

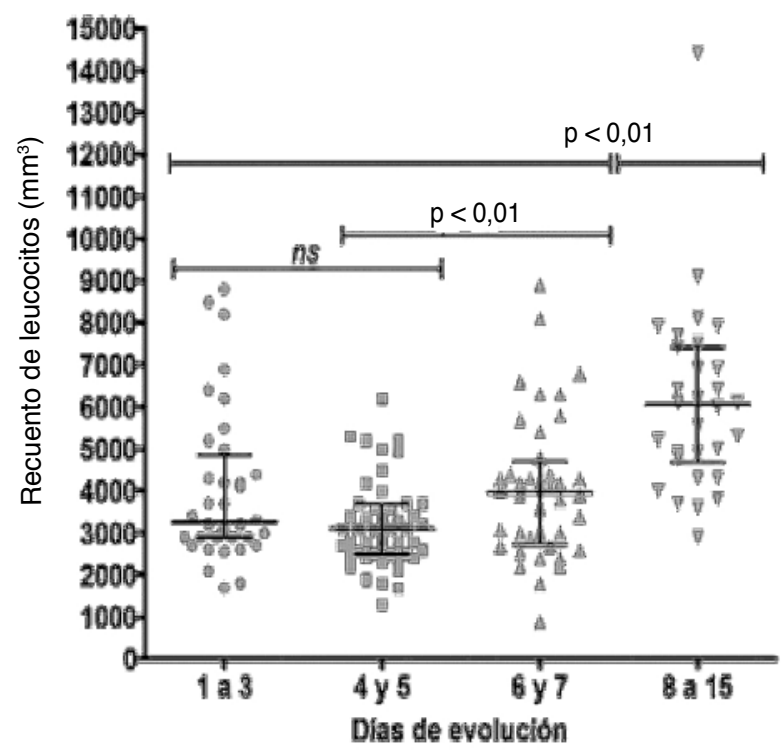

b.

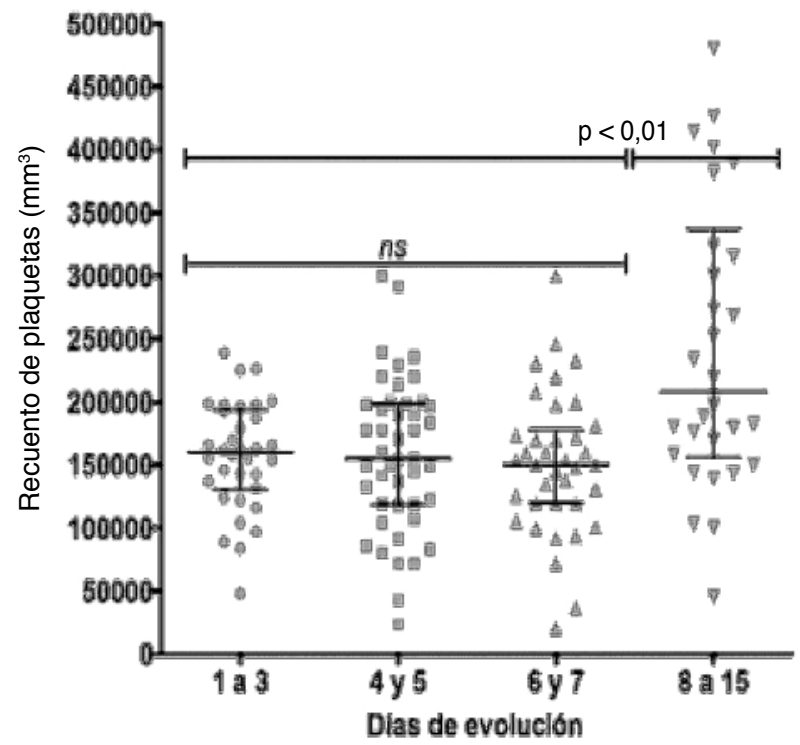

ns: no significativo.

a. Se encontró una diferencia estadísticamente significativa al comparar la media de leucocitos entre el día 4 y 5 y el día 6 y 7 ; lo mismo sucedió al comparar la media de leucocitos antes del día 7 y a partir del día 8 . Test de t, $\mathrm{p}<0,01$.

b. Se encontró una diferencia estadísticamente significativa al comparar el valor promedio de plaquetas antes del día 7 y a partir del día 8 . Test de $\mathrm{t}, \mathrm{p}<0,01$. 
(más importante en los primeros 5 días de enfermedad), la plaquetopenia y el aumento de transaminasas.

Dado que no existe un tratamiento específico, el reconocimiento precoz de los signos de alarma y el control hematológico resulta fundamental para detectar a los niños en riesgo y ofrecerles tratamiento de soporte en forma precoz. ${ }^{15}$

Según distintas publicaciones, el dengue sin signos de alarma ni comorbilidades podría tratarse en forma ambulatoria. En cuanto al dengue con comorbilidades, se recomienda un seguimiento ambulatorio estricto o internación en sala general. Si presenta signos de alarma, la recomendación es la internación en sala general y, en caso de dengue grave, el tratamiento deberá realizarse en la sala de Cuidados Intensivos. ${ }^{8}$ En esta serie, se siguieron estas recomendaciones, aunque, al inicio, se internaron pacientes con criterio entomológico.

Se conocen 4 serotipos virales. Los serotipos 2 y 3 se han asociado a casos más graves y mortalidad. La enfermedad genera una respuesta inmunitaria de tipo específico para cada serotipo (homóloga), que es duradera. Existe una inmunidad heteróloga hacia los demás serotipos que persiste por algunos meses y luego desaparece. ${ }^{15}$ Los serotipos 1 y 4 fueron los circulantes durante el brote presentado, según la OPS/OMS. En nuestro estudio, solo se constató la circulación del serotipo 1.

Las formas graves se presentan más frecuentemente durante la segunda infección; esto explicaría la falta de casos graves en nuestra serie, ya que nuestros pacientes se presentaron como primer caso de dengue 1 .

\section{REFERENCIAS}

1. Tittarelli E, LussoSB, GoyaS, et al.Dengue Virus 1 outbreak in Buenos Aires, Argentina, 2016. Emerg Infect Dis. 2017; 23(10):1684-5.
2. Argentina. Ministerio deSalud.Enfermedades infecciosas: Dengue. Guía para el equipo de salud. 4. ${ }^{a}$ ed. Buenos Aires: MISAL; 2015. [Consulta: 13 de junio de 2018]. Disponible en: http: / / www.msal.gob.ar/images/stories / bes / graficos / 0000000062cnt-guia-dengue-2016.pdf.

3. Shepard DS, Undurraga EA, Halasa YA, Stanaway JD. The global economic burden of dengue: a systematic analysis. Lancet Infect Dis. 2016; 16(8):935-41.

4. Grange L, Simon-Loriere E, Sakuntabhai A, et al. Epidemiological risk factors associated with high global frequency of inapparent dengue virus infections. Front Immunol. 2014; 5:280.

5. Tittarelli E, Barrero PR, Mistchenko AS, Valinotto LE. Secondary dengue virus infections during the 2009 outbreak in Buenos Aires. Trop Med Int Health. 2016; 21(1):28-32.

6. BhattS, Gething PW, Brady OJ, et al. The global distribution and burden of dengue. Nature. 2013; 496(446):504-7.

7. Pan American Health Organization, World Health Organization. Five-fold increase in dengue cases in the Americas over the past decade. 2014. [Consulta: 16 de noviembre de 2016]. Disponible en: http://www.paho. org $/ \mathrm{hq} /$ index.php?option $=$ com_content\&view $=$ article\& id=9657\&Itemid=1926\&lang=en.

8. Argentina. Ministerio de Salud. Boletín integrado de vigilancia. 2016;334,SE44. [Consulta: 10 de agosto de 2018]. Disponible en: https: / /www.argentina.gob.ar/salud/ epidemiologia/boletines2016.

9. World Health Organization. Dengue: guidelines for diagnosis, treatment, prevention and control. 2009. [Consulta:10 denoviembre de2016]. Disponible en: http: / / www.who.int/tdr/publications/documents / denguediagnosis.pdf?ua $=1$.

10. Morra ME, Altibi AMA, Igtadar S, et al. Definitions for warning signs and signs of severe dengue according to the WHO 2009 classification: Systematic review of literature. Rev Med Virol. 2018; 28(4):e1979.

11. Capeding MR, L'Azou M, Manalaysay M, et al. Laboratoryconfirmed Dengue in children in three regional hospitals in the Philippines in 2009-2010. Pediatr Infect Dis J. 2015; 34(11):1145-51.

12. Verhagen LM, de Groot R. Dengue in children. J Infect. 2014; 69(Suppl 1):S77-86.

13. Lovera D, Araya S, Mesquita MJ, et al. Prospective applicability study of the new dengue classification system for clinical management in children. Pediatr Infect Dis J. 2014; 33(9):933-5.

14. Azeredo EL, Dos Santos FB, Barbosa LS, et al. Clinical and Laboratory Profile of Zika and Dengue Infected Patients: Lessons Learned From the Co-circulation of Dengue, Zika and Chikungunya in Brazil. PLoS Curr. 2018; 10.

15. Kularatne SA. Dengue fever. BMJ. 2015; 351:h4661. 